\title{
The potential impact of pain on health outcomes among patients with chronic obstructive pulmonary disease
}

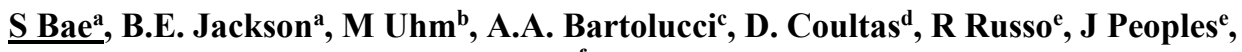 \\ J Ashmore ${ }^{f}$ and K.P. Singh ${ }^{a}$ \\ a. Division of Preventive Medicine, University of Alabama at Birmingham-Birmingham, AL/US \\ b. Daewon University, Jaecheon, ChungBuk/Korea \\ c. Department of Biostatistics, University of Alabama at Birmingham-Birmingham, AL/US \\ d. Veterans Affairs Portland Health Care System and Oregon Health Science University, Portland, \\ Oregon/US \\ e. University of Texas Health Science Center, Tyler-Tyler, TX/US \\ f. Baylor Medical Center, Behavioral Health Center, Plano, TX/US
}

Email: $\underline{\text { bsejong@uab.edu }}$

\begin{abstract}
:
Background

Pain among COPD patients is under-recognized and has not received in depth exploration. Our objective was to examine the relationship between patient-reported levels of pain with other characteristics to examine the associations with psychological distress, functional performance, and health care utilization.
\end{abstract}

\section{Methods}

This was a cross sectional analysis of baseline data from a randomized trial of physical activity selfmanagement. Pain was assessed using the Bodily Pain domain of the SF-12. Psychological distress was assessed with the SF-12 Mental composite score (MCS). Both SF-12 Scores range from 0 to 100; lower scores are suggestive of greater pain and greater psychological distress. Functional performance was assessed by 6 minute walk distance (6MW), Chronic Respiratory Questionnaire Dyspnea (CRQ). Healthcare utilization was based on self-reports for the 6 months prior to enrollment. Other patient characteristics included socio-demographics, body mass index (BMI) and spirometry. The distribution of pain was assessed and categorized into quartiles, we assessed for trends across pain quartiles using the Cochran-Armitage trend test for categorical variables and linear contrasts for continuous variables.

\section{Results}

Among the 325 patients with baseline data, we observed that the highest pain quartile was associated with younger age $(\mathrm{P}$-trend $=0.0003)$, higher BMI $(\mathrm{P}$-trend $=0.002)$, greater psychological distress $(\mathrm{P}$-trend $<0.0001)$, and less severe spirometric impairment $(\mathrm{P}$-trend=0.0004). In addition to this, we observed a large and clinically significant negative impact on CRQ dyspnea, 6MW distance, and health care utilization (P-trend $<0.01)$. Those who reported being in the high pain quartile more often reported hospitalizations and use of urgent care for lung and non-lung disease in the previous 6 months, as well as utilization of home health services.

\section{Conclusions}

Our findings suggest that pain has a large, clinically significant impact on psychological distress, functional performance and health care utilization. In order to improve the health outcomes of COPD patients reporting pain, effective interventions which enhance the recognition and management of pain are needed.

Keywords: Chronic obstructive pulmonary disease, health outcomes, quartile, pain 
Bae et al. The potential impact of pain on health outcomes among patients with chronic obstructive pulmonary disease

\section{INTRODUCTION}

Chronic Obstructive Pulmonary Disease (COPD) is a chronic progressive airway disease with rising morbidity and mortality (Viegi et al., 2007). While much research has examined environmental and behavioral factors associated with COPD, there has been a renewed focus on more patient centered outcomes (Celli et al., 2015). Chronic obstructive pulmonary disease (COPD) is the third leading cause of death in the United States, and is a larger contributor toward medical disability (Roberts, Mapel, \& Thomson, 2015). As the prevalence of this disease continues to increase over time, we still have much knowledge to gain regarding the improvement of COPD patient quality of life. A recent systematic review suggests that pain is a highly prevalent significant problem in patients with COPD (van Dam van Isselt et al., 2014). However, pain is an under-recognized symptom among patients with COPD and little is known about the health impact that pain may have on these patients (HajGhanbari, Garland, Road, \& Reid, 2013). In order to address this growing need, we aimed to examine the relationship between pain and indicators of health status including functional performance, quality of life, and health care utilization among patients with COPD.

\section{METHODS}

\section{a. Data Source}

This was a cross sectional analysis of baseline data collected from a pragmatic, self-management randomized clinical trial among patients with COPD designed to enhance daily lifestyle physical activity, which has previously described elsewhere (Ashmore et al., 2013; Russo et al., 2015). In brief, patients were enrolled from primary care and pulmonary specialty clinics of the University of Texas Health Science Center at Tyler. Once enrolled, patients filled out survey questionnaires to assess demographic information, measures of general and disease specific health, self-efficacy, and health care utilization. In addition to this, they also performed walking and step tests in order to gauge their physical activity levels and functional performance.

b. Study Population

Patients were eligible for this analysis if they were at least 45 years of age with physician-diagnosed COPD, and the ratio of post-bronchodilator Forced Expiratory Volume in 1 second (FEV $)_{1}$ to Forced Vital Capacity less than $70 \%$ and $\mathrm{FEV}_{1}$ less than $70 \%$.

c. Variables

The primary variable of interest in this analysis was pain, which was assessed using the Bodily Pain subdomain of the Medical Outcomes SF-12. Values ranged from 0 to 100, where higher scores indicate less pain. We also used the mental composite score (MCS) of the SF-12 as our measurement of psychological distress. Similar to the Bodily Pain subdomain, the range was from 0 to 100, where higher values suggest lower psychological distress. Based on their distribution, the pain scores were categorized according to quartiles. Functional performance was assessed by the self-paced 6-minute walk distance test (6MWD), and the Chronic Respiratory Questionnaire Dyspnea Domain (CRQD). Health care utilization was based on patient self-reports from the 6 months prior to their enrollment in the study. Health care utilization included hospitalization for lung and non-lung related diseases, urgent care for lung and non-lung related diseases, as well as any home health use. We also used demographic information obtained from the survey questionnaire including subjects age, annual income level $(\leq \$ 25 \mathrm{~K}$ and $>\$ 25 \mathrm{~K})$, Body Mass Index (BMI), and spirometry data. In addition to this we looked at various lifestyle and behavior related variables including smoking status, and pack years, the number of comorbid conditions as well as the Body mass index, airflow Obstruction, Dyspnea, and Exercise (BODE) score.

\section{d. Data analysis}

The distribution of patient characteristics and health outcomes were presented overall and across the quartiles of the bodily pain distribution. Means and standard deviations (SD) were calculated for continuous variables, and frequency and percentages were calculated for categorical variables. We assessed for trends in characteristics and health outcomes across all 4 pain quartiles using the Cochran-Armitage trend test for categorical variables and ANOVA for normally distributed continuous variables, and Kruskal Wallis test for medians. Moreover testing for linear trends for medians were assessed by the Jonckheer-Terpstra test 
Bae et al. The potential impact of pain on health outcomes among patients with chronic obstructive pulmonary disease

(Jonckheere AR, 1954). Data were analyzed using SAS v9.4, all results were considered statistically significant at the 0.05 level.

\section{RESULTS}

There were 325 patients with baseline data (Table 1), of which the average age was 68 years of age, where $50.46 \%$ were female, predominantly non-Hispanic White (91.69\%), Married (56.9\%), Retired (60.49\%), and Less than $\$ 25 \mathrm{~K}$ annual income (58.68\%). Approximately $2 / 3$ rds of the sample were ex-smokers $(67.38 \%)$, with a mean (SD) pack years of 58.77(36.47). Subjects reported a median of 3 comorbidities, and most did not have depressive symptoms (73.23\%). The average BMI was 28.98, and most patients were classified as GOLD stage II and III (85.84\%).

There was an observed linear trend across pain quartiles for patient age $(p=0.0003)$, where patients with the least amount of self-reported pain were older than those with the most amount of pain. Moreover there were education $(\mathrm{p}=0.0163)$ and income $(\mathrm{p}=0.0006)$ differences across the pain quartiles, where a greater proportion of subjects with the most amount of pain had high school or less education and less than $\$ 25 \mathrm{~K}$ annual income compared to those with the least amount of pain. The proportion of subjects who were classified as "disabled" was greatest among those in the highest pain quartile (49.4\%), which was statistically different $(\mathrm{p}<0.0001)$ than the distribution in the other lower pain quartiles. In terms of patient health status and behaviors (Table 2) tests for linear trend suggested that patients in the highest pain quartile had a greater number of pack years $(\mathrm{p}=0.05)$, more co-morbid conditions $(\mathrm{p}=0.0019)$, higher $\mathrm{BMI}(\mathrm{p}=0.002)$, and higher FEV1 ( $p=0.0004)$, higher MMRC Dyspnea $(p=0.0003)$, and a lower proportion which could walk greater than $350 \mathrm{~m}$ in the 6 minute walk ( $\mathrm{p}=0.0028$ ) compared to those with the lowest pain quartile. Moreover, when looking at the Geriatric Depression Scale, we see that the proportion of subjects reporting moderate to severe depressive symptoms are greatest within the highest pain quartile $(51.1 \%)$, which is almost double that of the next highest pain quartile $(26.7 \%)$, and nearly 4 times that of the lowest pain quartile $(12.0 \%)$.

Table 3 displays the health outcome measures and healthcare utilization of the subjects both overall and across pain quartiles. On average the mean (SD) 6MW distance was 338.17 (96.8) meters, and for the Mental Composite Score 50.39 (11.15). Within the previous 6 months, it appears that more than 2/3rds of the sample had made visits to a medical office to see a physician, nurse, nurse practitioner, or physician's assistant for their lung or non-lung related diseases. Approximately $15 \%$ had been either hospitalized for lung disease, been to urgent care for non-lung disease and were not hospitalized. There were statistically significant linear trends across health outcome measures, where patients in the highest pain quartile had lower dyspnea $(\mathrm{p}<0.0001)$, shorter 6MW distance $(\mathrm{p}=0.0016)$, and lower MCS scores $(\mathrm{p}<0.0001)$. The proportion of health care utilization was greater among those in the highest pain quartile compared to the lowest pain quartile. Where in the previous 6 months, there were statistically significant linear trends across pain quartiles for having been hospitalized for lung disease $(p=0.0179)$, non-lung disease $(p=0.0035)$, having been to urgent care or the emergency room but not hospitalized for lung disease $(p=0.0108)$ and non-lung disease $(\mathrm{p}=0.0199)$, and having used any home health services $(\mathrm{p}=0.0051)$.

\section{DISCUSSION AND CONCLUSIONS}

Our overall and subgroup-specific results suggest that demographic factors including age, education, and income may be associated with self-reported pain. It appears that patients in the least pain quartile are older than those in the most pain quartile; this may be due to older patients having dealt with COPD-related pain and received treatment or developed coping mechanisms. Education and income both appear to be associated with perceived pain, whereas those with more pain were more likely to have lower education and income. In terms of health status and behavior, higher average pack years of smoking cigarettes were associated with higher pain quartile. Similarly, having a greater number of comorbidities was associated with greater pain, as well as BMI, FEV1, self-reported dyspnea. The Dyspnea, Fatigue, and Emotional Functioning domains of the Chronic Respiratory Questionnaire all had significant results for linear tests for trend, where the most pain quartile had lower scores that those with the least amount of pain. Lower pain was associated with further 
Bae et al. The potential impact of pain on health outcomes among patients with chronic obstructive pulmonary disease

6MW distance and higher mental quality of life as measured by the SF-12, similar to what has been observed across multiple pain related studies in the literature (Lee, Harrison, Goldstein, \& Brooks, 2015).

The higher proportion of younger and disabled individuals with suggestion of moderate to severe depressive symptoms presents an interesting finding. These are the individuals with the highest pain, comorbidity counts, and generally highest health care utilization who are still relatively young among our COPD population. The presence of additional comorbidities along with COPD, can affect psychological well-being leading to depression which may be drawn out over the life course for these individuals, which in turn may affect their healthcare utilization (Sabbatini, Nallamothu, \& Kocher, 2014). These patients represent a subset of those whom would benefit greatly from future interventions targeting pain management and coping strategies.

One consideration when interpreting our results is that the assessment of pain was from a subdomain of the SF-12. While this provides a starting point for further analyses, this was a secondary analysis of this trial's data which was not specifically designed to measure pain. With that in mind, we did observe differences across pain strata which can be used as a platform for other future research. While this assessment was cross sectional in nature, it is unclear if the pain reported was COPD related or had been an existing pain from a comorbid condition. Moreover the type and location of pain was not known, leg pain may have a different association with health outcomes than chest pain among the COPD population.

In summary, our findings suggest that among COPD patients, pain has a large and clinically significant impact on psychological distress, functional performance, and healthcare utilization. Future studies which seek to improve the health outcomes of COPD patients reporting pain, should consider enhancing the recognition and management of pain. Research should be conducted with validated measures of pain in order to help understand any causal link which could further benefit the effectiveness of interventions.

\section{ACKNOWLEDGEMENTS}

The authors would like to acknowledge the study participants and the research staff members, as well as the staff of the University of Texas Health Science Center-Tyler Cardiopulmonary Services. This analysis was funded in part by a grant from the National Institutes of Health (NHLBI R18HL092955).

\section{REFERENCES}

Ashmore, J., Russo, R., Peoples, J., Sloan, J., Jackson, B. E., Bae, S., et al. (2013). Chronic obstructive pulmonary disease self-management activation research trial (COPD-SMART): Design and methods. Contemp Clin Trials, 35(2), 77-86.

Celli, B. R., Decramer, M., Wedzicha, J. A., Wilson, K. C., Agusti, A. A., Criner, G. J., et al. Research, A. E. T. F. f. C. (2015). An official American Thoracic Society/European Respiratory Society statement: research questions in COPD. Eur Respir Rev, 24(136), 159-172.

HajGhanbari, B., Garland, S. J., Road, J. D., and Reid, W. D. (2013). Pain and physical performance in people with COPD. Respir Med, 107(11), 1692-1699.

Jonckheere AR. (1954). A Distribution-Free K-sample Test Against Ordered Alternatives. Biometrika, 41(1/2), 133-135.

Lee, A. L., Harrison, S. L., Goldstein, R. S., and Brooks, D. (2015). Pain and its clinical associations in individuals with COPD: a systematic review. Chest, 147(5), 1246-1258. doi: 10.1378/chest.14-2690

Roberts, M. H., Mapel, D. W., and Thomson, H. N. (2015). The impact of chronic pain on direct medical utilization and costs in chronic obstructive pulmonary disease. Clinicoecon Outcomes Res, 7, 173-184.

Russo, R., Coultas, D., Ashmore, J., Peoples, J., Sloan, J., Jackson, B. E., et al.. (2015). Chronic obstructive pulmonary disease self-management activation research trial (COPD-SMART): results of recruitment and baseline patient characteristics. Contemp Clin Trials, 41, 192-201.

Sabbatini, A. K., Nallamothu, B. K., and Kocher, K. E. (2014). Reducing variation in hospital admissions from the emergency department for low-mortality conditions may produce savings. Health Aff (Millwood), 33(9), 1655-1663.

van Dam van Isselt, E. F., Groenewegen-Sipkema, K. H., Spruit-van Eijk, M., Chavannes, N. H., de Waal, M. W., Janssen, D. J., and Achterberg, W. P. (2014). Pain in patients with COPD: a systematic review and meta-analysis. BMJ Open, 4(9),

Viegi, G., Pistelli, F., Sherrill, D. L., Maio, S., Baldacci, S., and Carrozzi, L. (2007). Definition, epidemiology and natural history of COPD. Eur Respir J, 30(5), 993-1013. 
Table 1. Characteristics of subjects stratified by Bodily Pain Quartiles

\begin{tabular}{|c|c|c|c|c|c|c|c|c|c|c|c|c|}
\hline \multirow[b]{2}{*}{ Variables } & \multirow{2}{*}{$\begin{array}{l}\text { Overall } \\
\mathbf{N}=\mathbf{3 2 5}\end{array}$} & & \multicolumn{2}{|c|}{$\begin{array}{c}\text { Most Pain } \\
\text { Bodily Pain Q1 }\end{array}$} & \multicolumn{2}{|c|}{ Bodily Pain Q2 } & \multicolumn{2}{|c|}{ Bodily Pain Q3 } & \multicolumn{2}{|c|}{$\begin{array}{c}\text { Least pain } \\
\text { Bodily Pain Q4 }\end{array}$} & \multirow{2}{*}{$\begin{array}{l}\text { Overall } \\
\text { P-value }\end{array}$} & \multirow{2}{*}{$\begin{array}{c}\text { Test } \\
\text { for } \\
\text { Trend } \\
\text { P-value } \\
\end{array}$} \\
\hline & & & $\mathrm{N}=86$ & & $\mathrm{~N}=90$ & & $\mathrm{~N}=74$ & & $\mathrm{~N}=75$ & & & \\
\hline Age (years), mean (SD) & 68.47 & 9.5 & 64.92 & 9.6 & 68.99 & 9.7 & 70.33 & 8.8 & 70.08 & 8.7 & 0.0005 & 0.0003 \\
\hline Gender, $\mathrm{n}(\%)$ & & & & & & & & & & & 0.4346 & 0.144 \\
\hline Male & 161 & 49.5 & 38 & 44.2 & 42 & 46.7 & 41 & 55.4 & 40 & 53.3 & & \\
\hline Female & 164 & 50.5 & 48 & 55.8 & 48 & 53.3 & 33 & 44.6 & 35 & 46.7 & & \\
\hline Race/Ethnicity, n(\%) & & & & & & & & & & & & -- \\
\hline NH White & 298 & 91.7 & 77 & 89.5 & 81 & 90.0 & 67 & 90.5 & 73 & 97.3 & & \\
\hline NH Black & 22 & 6.8 & 8 & 9.3 & 6 & 6.7 & 6 & 8.1 & 2 & 2.7 & & \\
\hline Other & 5 & 1.5 & 1 & 1.2 & 2 & 3.3 & 1 & 1.4 & 0 & 0.0 & & \\
\hline Marital Status, n(\%) & & & & & & & & & & & 0.1081 & -- \\
\hline Single & 30 & 9.2 & 13 & 15.1 & 7 & 7.8 & 5 & 6.8 & 5 & 6.7 & & \\
\hline Married & 185 & 56.9 & 38 & 44.2 & 57 & 63.3 & 43 & 58.1 & 47 & 62.7 & & \\
\hline Widowed & 61 & 18.8 & 18 & 20.9 & 19 & 21.1 & 14 & 18.9 & 10 & 13.3 & & \\
\hline Separated & 49 & 15.1 & 17 & 19.8 & 7 & 7.8 & 12 & 16.2 & 13 & 17.3 & & \\
\hline Work Status, n(\%) & & & & & & & & & & & $<0.0001$ & -- \\
\hline Disabled & 79 & 24.4 & 43 & 49.4 & 17 & 19.1 & 12 & 16.2 & 7 & 9.5 & & \\
\hline Retired & 196 & 60.5 & 36 & 41.4 & 58 & 65.2 & 50 & 67.6 & 52 & 70.3 & & \\
\hline Other & 49 & 15.1 & 8 & 9.2 & 14 & 15.7 & 12 & 16.2 & 15 & 20.3 & & \\
\hline Education, $\mathrm{n}(\%)$ & & & & & & & & & & & 0.0525 & 0.0163 \\
\hline Highschool or Less & 165 & 50.8 & 52 & 60.5 & 49 & 54.4 & 30 & 40.5 & 34 & 45.3 & & \\
\hline More than Highschool & 160 & 49.2 & 34 & 39.5 & 41 & 45.6 & 44 & 59.5 & 41 & 54.7 & & \\
\hline Income Level, n(\%) & & & & & & & & & & & 0.0012 & 0.0006 \\
\hline LE $25 \mathrm{~K}$ & 186 & 58.7 & 65 & 76.5 & 49 & 55.7 & 35 & 49.3 & 37 & 50.7 & & \\
\hline GT $25 \mathrm{~K}$ & 131 & 41.3 & 20 & 23.5 & 39 & 44.3 & 36 & 50.7 & 36 & 49.3 & & \\
\hline Rural & 158 & 48.8 & 41 & 47.7 & 43 & 47.8 & 39 & 52.7 & 35 & 47.3 & 0.8966 & 0.8664 \\
\hline
\end{tabular}

Test for differences using ANOVA for continuous variables, Kruskal-Wallis test for medians, and chi-square or Fisher's exact test for categorical variables;

Test for linear trend using Cochrane-Armitage trend test for categorical variables, and linear contrasts for continuous variables 
Table 2. Patient Health Status and Behaviors (N=325)

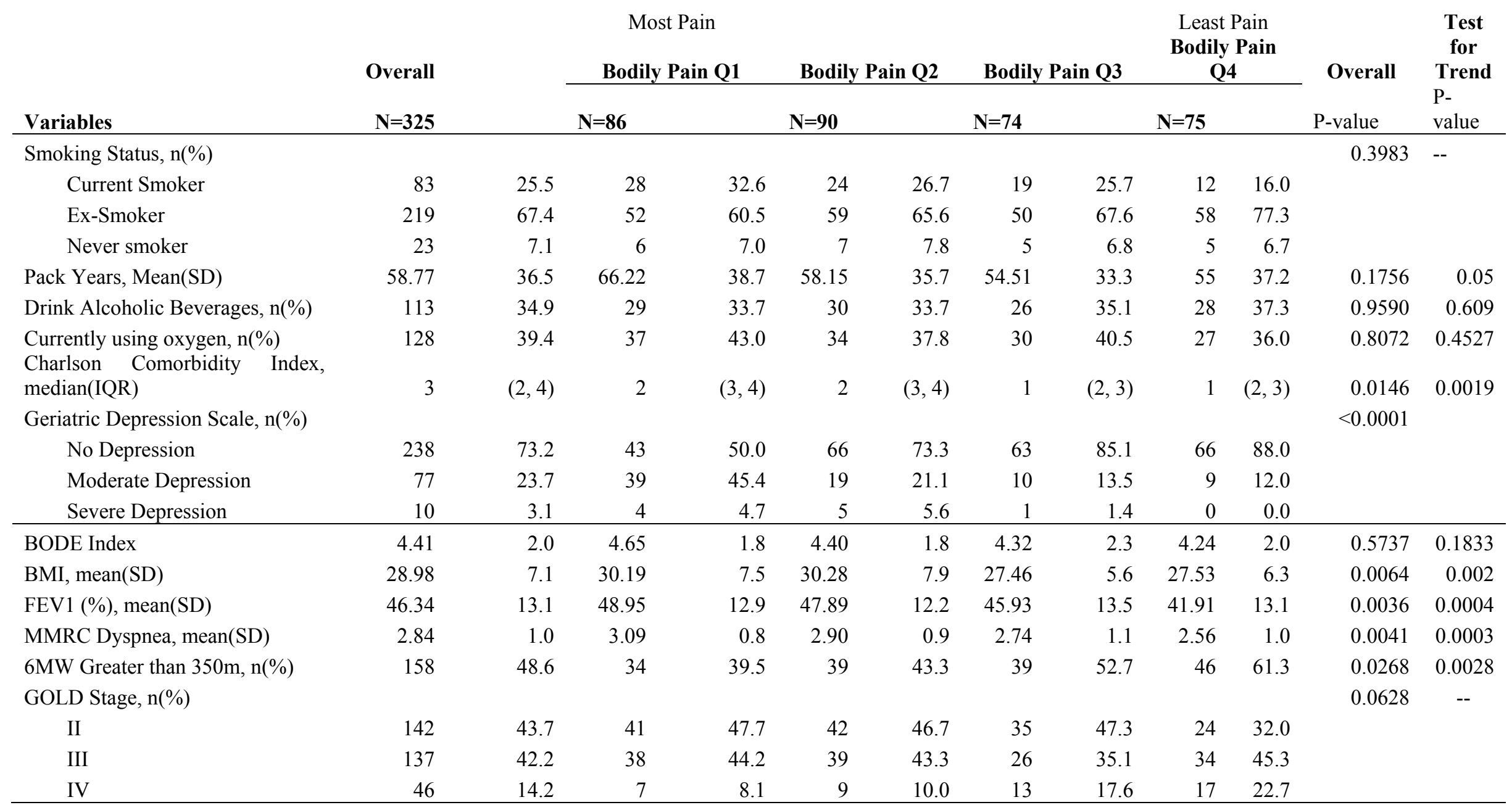

BODE-Body Mass Index, Degree of Airflow Obstruction and Dyspnea, and Exercise Capacity; Test for differences using ANOVA for continuous variables, Kruskal-Wallis test for medians, and chi-square or Fisher's exact test for categorical variables Test for linear trend using Cochrane-Armitage trend test for categorical variables, linear contrasts for continuous variables, and Jonckheer-Terpstra test for medians 
Table 3. Health Outcome Measures of Randomized subjects ( $\mathrm{N}=325)$

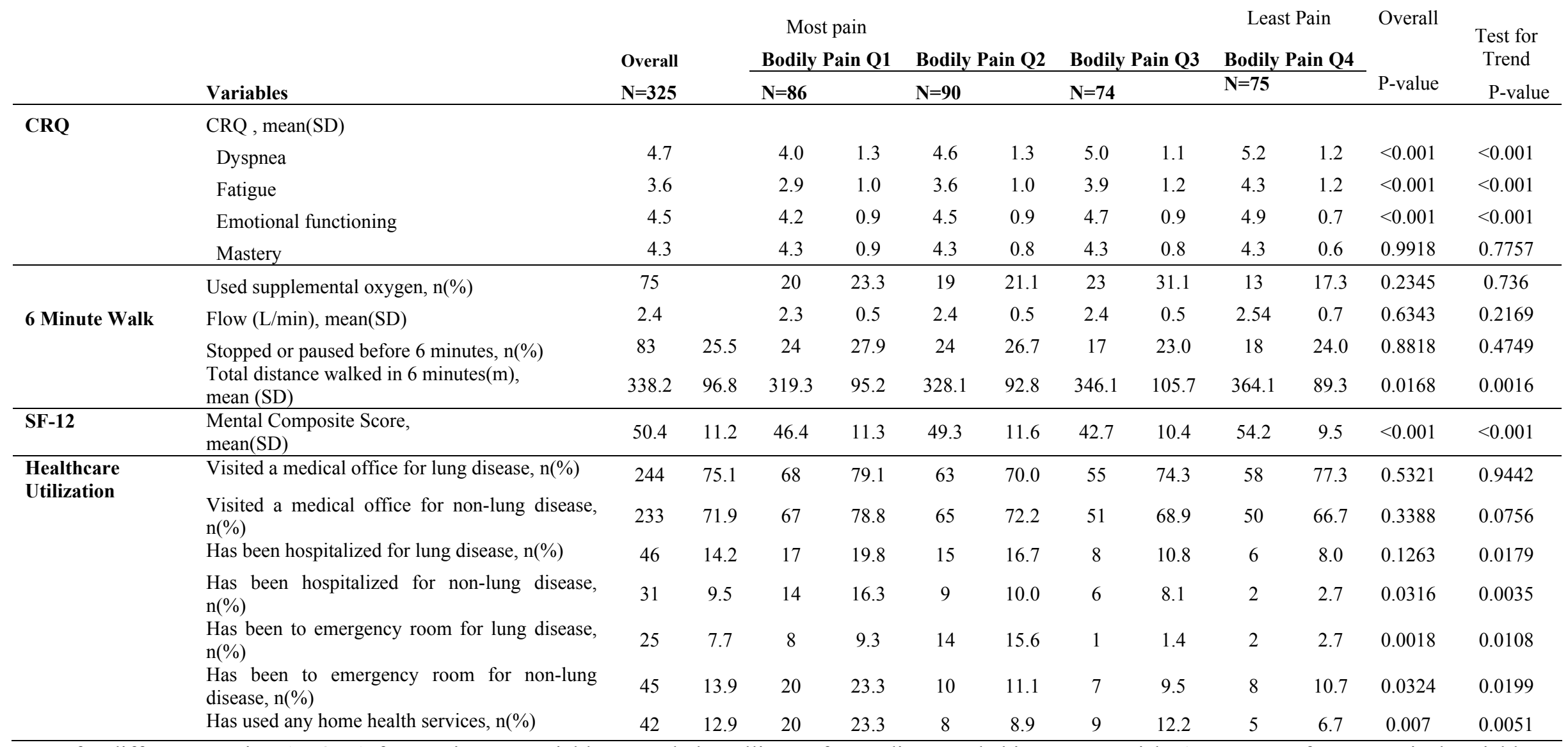

Test for differences using ANOVA for continuous variables, Kruskal-Wallis test for medians, and chi-square or Fisher's exact test for categorical variables

Test for linear trend using Cochrane-Armitage trend test for categorical variables, and linear contrasts for continuous variables 